\title{
Solid-state NMR reveals the structural transformation of the TDP-43 amyloidogenic region upon fibrillation
}

\author{
Xiao-Feng Zhuo\#+®, Jian Wang\#, Jing Zhang\#+®, Lei-Lei Jiang + , Hong-Yu Hu*+ and \\ Jun-Xia Lu*\# \\ \#School of Life Science and Technology, ShanghaiTech University, Shanghai 201210, P. R. China \\ † State Key Laboratory of Molecular Biology, CAS Center for Excellence in Molecular Cell Science, Shanghai \\ Institute of Biochemistry and Cell Biology, Chinese Academy of Sciences, Shanghai 200031, P. R. China \\ $\otimes$ University of Chinese Academy of Sciences, Beijing 100049, P. R. China
}


Table S1. MAS NMR assignments for the TDP-43 (311-360) amyloid fibrils

\begin{tabular}{|c|c|c|c|c|c|c|c|c|c|c|c|c|c|c|c|}
\hline Res & Res & $\mathrm{N}$ & $\mathrm{CO}$ & $\mathrm{CA}$ & $\mathrm{CB}$ & CG & CG1 & CG2 & $\mathrm{CD}$ & $\mathrm{CD} 1$ & $\mathrm{CD} 2$ & $\mathrm{CE}$ & $\mathrm{CZ}$ & $\mathrm{Nd} / \mathrm{N} \varepsilon$ & $\mathrm{H}^{\mathrm{N}}$ \\
\hline Number & type & & & & & & & & & & & & & & \\
\hline 311 & $\mathrm{M}$ & & & & & & & & & & & & & & \\
\hline 312 & $\mathrm{~N}$ & & & & & & & & & & & & & & \\
\hline 313 & $\mathrm{~F}$ & 120.6 & 175.9 & 57.0 & 44.3 & & & & 130.3 & & & & & & \\
\hline 314 & $\mathrm{G}$ & 102.6 & 172.9 & 44.7 & & & & & & & & & & & 6.61 \\
\hline 315 & A & 120.4 & 172.8 & 52.6 & 19.2 & & & & & & & & & & \\
\hline 316 & $\mathrm{~F}$ & 122.2 & 174.8 & 56.7 & 43.3 & 137.6 & & & 131.9 & & & 130.5 & & & 9.17 \\
\hline 317 & S & 126.3 & 173.3 & 56.7 & 67.1 & & & & & & & & & & 8.97 \\
\hline 318 & I & 121.8 & 172.6 & 61.5 & 39.3 & & 26.6 & 17.9 & & 14.2 & & & & & 7.75 \\
\hline 319 & $\mathrm{~N}$ & 118.8 & 172.8 & 48.6 & 37.9 & 176.7 & & & & & & & & 109.6 & 8.08 \\
\hline 320 & $\mathrm{P}$ & 133.2 & 174.8 & 62.1 & 33.3 & 28.2 & & & 49.5 & & & & & & \\
\hline 321 & A & 123.1 & 174.8 & 50.6 & 21.5 & & & & & & & & & & 8.73 \\
\hline 322 & $\mathrm{M}$ & 124.5 & 174.5 & 54.7 & 34.2 & 31.8 & & & & & & 17.9 & & & \\
\hline 323 & $\mathrm{M}$ & & & & & & & & & & & & & & \\
\hline 324 & A & 126.9 & 175.8 & 50.9 & 16.2 & & & & & & & & & & \\
\hline 325 & A & 117.6 & 174.5 & 49.9 & 25.4 & & & & & & & & & & 8.14 \\
\hline 326 & A & 117.8 & 176.6 & 49.9 & 23.1 & & & & & & & & & & 8.23 \\
\hline 327 & Q & 119.3 & 176.2 & 60.3 & 28.2 & 34.8 & & & 177.2 & & & & & 108.6 & 7.73 \\
\hline 328 & A & 119.8 & 173.8 & 50.1 & 21.7 & & & & & & & & & & 8.70 \\
\hline 329 & A & 127.4 & 174.4 & 49.9 & 20.5 & & & & & & & & & & 9.56 \\
\hline 330 & $\mathrm{~L}$ & 127.5 & 175.2 & 53.8 & 43.5 & 28.8 & & & & 27.1 & & & & & 9.49 \\
\hline 331 & Q & 122.4 & 175.2 & 56.5 & 26.7 & 34.7 & & & 180.6 & & & & & 110.8 & \\
\hline 332 & $S$ & 113.7 & 171.9 & 58.5 & 68.1 & & & & & & & & & & 7.97 \\
\hline 333 & S & 114.2 & 172.0 & 56.5 & 66.5 & & & & & & & & & & 7.97 \\
\hline 334 & $\mathrm{~W}$ & 125.4 & & 58.4 & 33.9 & 113.4 & & & & 124.7 & 129.4 & 138.2 & 113.8 & 128.9 & \\
\hline 335 & G & & & & & & & & & & & & & & \\
\hline 336 & $M$ & & & & & & & & & & & & & & \\
\hline 337 & $\mathrm{M}$ & & & & & & & & & & & & & & \\
\hline 338 & $\mathrm{G}$ & & & & & & & & & & & & & & \\
\hline 339 & $\mathrm{M}$ & & & & & & & & & & & & & & \\
\hline 340 & $\mathrm{~L}$ & & & & & & & & & & & & & & \\
\hline 341 & $\mathrm{~A}$ & & & & & & & & & & & & & & \\
\hline 342 & $S$ & & & & & & & & & & & & & & \\
\hline 343 & Q & 123.4 & 174.8 & 54.5 & 34.2 & 36.4 & & & 179.7 & & & & & 113.2 & 8.69 \\
\hline 344 & Q & 126.8 & 174.4 & 54.9 & 29.9 & 34.1 & & & 176.9 & & & & & 114.2 & 7.79 \\
\hline 345 & $\mathrm{~N}$ & 124.1 & 175.7 & 50.8 & 39.7 & 175.4 & & & & & & & & 107.0 & 8.55 \\
\hline 346 & Q & 115.1 & 177.9 & 56.5 & 26.6 & 35.6 & & & 181.1 & & & & & 111.7 & 8.73 \\
\hline 347 & S & 115.6 & 174.7 & 58.3 & 66.3 & & & & & & & & & & 8.44 \\
\hline 348 & G & 109.9 & 170.8 & 44.1 & & & & & & & & & & & 8.94 \\
\hline 349 & $\mathrm{P}$ & 132.3 & 176.7 & 61.6 & 31.6 & 27.5 & & & 48.9 & & & & & & \\
\hline
\end{tabular}




$\begin{array}{llllllll}350 & \mathrm{~S} & 123.4 & 174.9 & 55.5 & 65.5 & & \\ 351 & \mathrm{G} & 110.8 & 174.5 & 47.5 & & & \\ 352 & \mathrm{~N} & 116.5 & 174.5 & 53.3 & 40.5 & & \\ 353 & \mathrm{~N} & 120.9 & 174.8 & 53.1 & 42.1 & 177.0 & 6.86 \\ 354 & \mathrm{Q} & 123.5 & & 54.7 & 31.6 & 38.2 & 9.28 \\ 355 & \mathrm{~N} & 123.4 & 174.5 & 52.7 & 41.3 & 176.8 & 9.16 \\ 356 & \mathrm{Q} & & & & & & \\ 357 & \mathrm{G} & & & & & & \\ 358 & \mathrm{~N} & & & & & & \\ 359 & \mathrm{M} & & & & \end{array}$

Table S2. TALOS-N prediction of the secondary structure of the TDP-43 (311-360) fibrils from assigned chemical shifts.

\begin{tabular}{|c|c|c|c|c|c|c|}
\hline Res Number & Res type & Prob a-helix & Prob b-strand & Prob loop & Confidence & Secondary Structure \\
\hline 311 & M & 0 & 0 & 0 & 0 & $\mathrm{X}$ \\
\hline 312 & $\mathrm{~N}$ & 0 & 0 & 0 & 0 & $\mathrm{x}$ \\
\hline 313 & $\mathrm{~F}$ & 0.333 & 0.333 & 0.333 & 0 & $\mathrm{~L}$ \\
\hline 314 & G & 0.032 & 0.446 & 0.522 & 0.08 & $\mathrm{~L}$ \\
\hline 315 & A & 0.024 & 0.849 & 0.126 & 0.72 & E \\
\hline 316 & F & 0.036 & 0.935 & 0.029 & 0.90 & $\mathrm{E}$ \\
\hline 317 & S & 0.001 & 0.905 & 0.094 & 0.81 & $\mathrm{E}$ \\
\hline 318 & I & 0.000 & 0.482 & 0.518 & 0.04 & $\mathrm{~L}$ \\
\hline 319 & $\mathrm{~N}$ & 0.001 & 0.153 & 0.846 & 0.69 & $\mathrm{~L}$ \\
\hline 320 & $\mathrm{P}$ & 0.015 & 0.349 & 0.636 & 0.29 & $\mathrm{~L}$ \\
\hline 321 & A & 0.000 & 0.862 & 0.138 & 0.72 & $\mathrm{E}$ \\
\hline 322 & M & 0.000 & 0.972 & 0.028 & 0.94 & $\mathrm{E}$ \\
\hline 323 & M & 0.020 & 0.955 & 0.025 & 0.93 & E \\
\hline 324 & A & 0.000 & 0.803 & 0.197 & 0.61 & $\mathrm{E}$ \\
\hline 325 & A & 0.000 & 0.900 & 0.100 & 0.80 & E \\
\hline 326 & A & 0.008 & 0.691 & 0.301 & 0.39 & E \\
\hline 327 & Q & 0.016 & 0.245 & 0.739 & 0.49 & $\mathrm{~L}$ \\
\hline 328 & A & 0.016 & 0.397 & 0.586 & 0.19 & $\mathrm{~L}$ \\
\hline 329 & A & 0 & 0.852 & 0.148 & 0.7 & E \\
\hline 330 & $\mathrm{~L}$ & 0.002 & 0.937 & 0.061 & 0.88 & E \\
\hline 331 & Q & 0.009 & 0.644 & 0.348 & 0.3 & E \\
\hline 332 & $S$ & 0.024 & 0.582 & 0.393 & 0.19 & E \\
\hline 333 & $S$ & 0.041 & 0.865 & 0.094 & 0.77 & E \\
\hline 334 & W & 0.1 & 0.832 & 0.068 & 0.73 & E \\
\hline 335 & G & 0.426 & 0.182 & 0.392 & 0.03 & $X$ \\
\hline 336 & $\mathrm{M}$ & 0.596 & 0.08 & 0.323 & 0.27 & $\mathrm{X}$ \\
\hline 337 & $\mathrm{M}$ & 0.586 & 0.1 & 0.315 & 0.27 & $\mathrm{X}$ \\
\hline
\end{tabular}




\begin{tabular}{|c|c|c|c|c|c|c|}
\hline 338 & G & 0.578 & 0.208 & 0.214 & 0.36 & $\mathrm{X}$ \\
\hline 339 & $\mathrm{M}$ & 0.543 & 0.29 & 0.168 & 0.25 & $\mathrm{X}$ \\
\hline 340 & $\mathrm{~L}$ & 0.474 & 0.262 & 0.265 & 0.21 & $\mathrm{X}$ \\
\hline 341 & $\mathrm{~A}$ & 0.458 & 0.264 & 0.278 & 0.18 & $\mathrm{X}$ \\
\hline 342 & $S$ & 0.005 & 0.872 & 0.123 & 0.75 & $\mathrm{E}$ \\
\hline 343 & Q & 0.018 & 0.954 & 0.029 & 0.92 & $\mathrm{E}$ \\
\hline 344 & Q & 0.081 & 0.86 & 0.06 & 0.78 & $\mathrm{E}$ \\
\hline 345 & $\mathrm{~N}$ & 0.002 & 0.223 & 0.775 & 0.55 & $\mathrm{~L}$ \\
\hline 346 & Q & 0.036 & 0.077 & 0.887 & 0.81 & $\mathrm{~L}$ \\
\hline 347 & $S$ & 0.041 & 0.044 & 0.915 & 0.87 & $\mathrm{~L}$ \\
\hline 348 & G & 0.027 & 0.122 & 0.851 & 0.73 & $\mathrm{~L}$ \\
\hline 349 & $\mathrm{P}$ & 0 & 0.293 & 0.707 & 0.41 & $\mathrm{~L}$ \\
\hline 350 & $\mathrm{~S}$ & 0.021 & 0.1 & 0.879 & 0.78 & $\mathrm{~L}$ \\
\hline 351 & G & 0.01 & 0.054 & 0.937 & 0.88 & $\mathrm{~L}$ \\
\hline 352 & $\mathrm{~N}$ & 0.014 & 0.047 & 0.939 & 0.89 & $\mathrm{~L}$ \\
\hline 353 & $\mathrm{~N}$ & 0.044 & 0.225 & 0.731 & 0.51 & $\mathrm{~L}$ \\
\hline 354 & Q & 0 & 0.756 & 0.244 & 0.51 & $\mathrm{E}$ \\
\hline 355 & $\mathrm{~N}$ & 0.03 & 0.709 & 0.261 & 0.45 & $\mathrm{E}$ \\
\hline 356 & Q & 0.094 & 0.328 & 0.577 & 0.25 & $\mathrm{X}$ \\
\hline 357 & G & 0 & 0.464 & 0.536 & 0.07 & $\mathrm{X}$ \\
\hline 358 & $\mathrm{~N}$ & 0.024 & 0.322 & 0.654 & 0.33 & $\mathrm{X}$ \\
\hline 359 & M & 0 & 0.109 & 0.891 & 0.78 & $\mathrm{X}$ \\
\hline 360 & $Q$ & 0.013 & 0 & 0.987 & 0.97 & $X$ \\
\hline
\end{tabular}

Figure S1. Sequence of Trx-TDP-43(311-360) with the residues 311-360 highlighted in red. The purple indicates two extra 
residues from enzyme cleavage.

MSDKIIHLTDDSFDTDVLKADGAILVDFWAEWCGPCKMIAPILDEIADEYQGKLTVAKLNIDQNPGTAPKYGIRGI PTLLLFKNGEVAATKVGALSKGQLKEFLDANLAGSGSGHMHHHHHHSSGLVPRGSMNFGAFSINPAMMAAAQA ALQSSWGMMGMLASQQNQSGPSGNNQNQGNMQ

Figure S2. LLPS of TDP-43(311-360)-GFP at a concentration of $26 \mu \mathrm{M}$. The sample was kept in a buffer (50 mM Tris-HCl, $300 \mathrm{mM} \mathrm{NaCl}, 500 \mathrm{mM}$ Imidazole, $\mathrm{pH} 8.0$ ) and on a water bath at $25^{\circ} \mathrm{C}$ for different intervals before imaging. (a) and (b) without torula yeast RNA; (c) and (d) with additional torula yeast RNA. (a) DIC and TEM images of samples after $2 \mathrm{~h}$ incubation, indicating little liquid droplets formation in DIC image and no large particles observed under TEM. (b) DIC and TEM images of samples after $16 \mathrm{~h}$ incubation, showing liquid droplets in DIC and amorphous protein aggregates under TEM. (c) DIC and TEM images of samples after $2 \mathrm{~h}$ incubation, showing liquid droplets in DIC image (selected droplets were shown in expansion at the bottom as labeled) and amorphous aggregates observed under TEM. (d) DIC and TEM images of samples after $16 \mathrm{~h}$ incubation, showing larger liquid droplets in DIC, amorphous aggregates and fibrils under TEM. The scale bar of the microscopic images is $20 \mu \mathrm{m}$ and that of is $500 \mathrm{~nm}$.

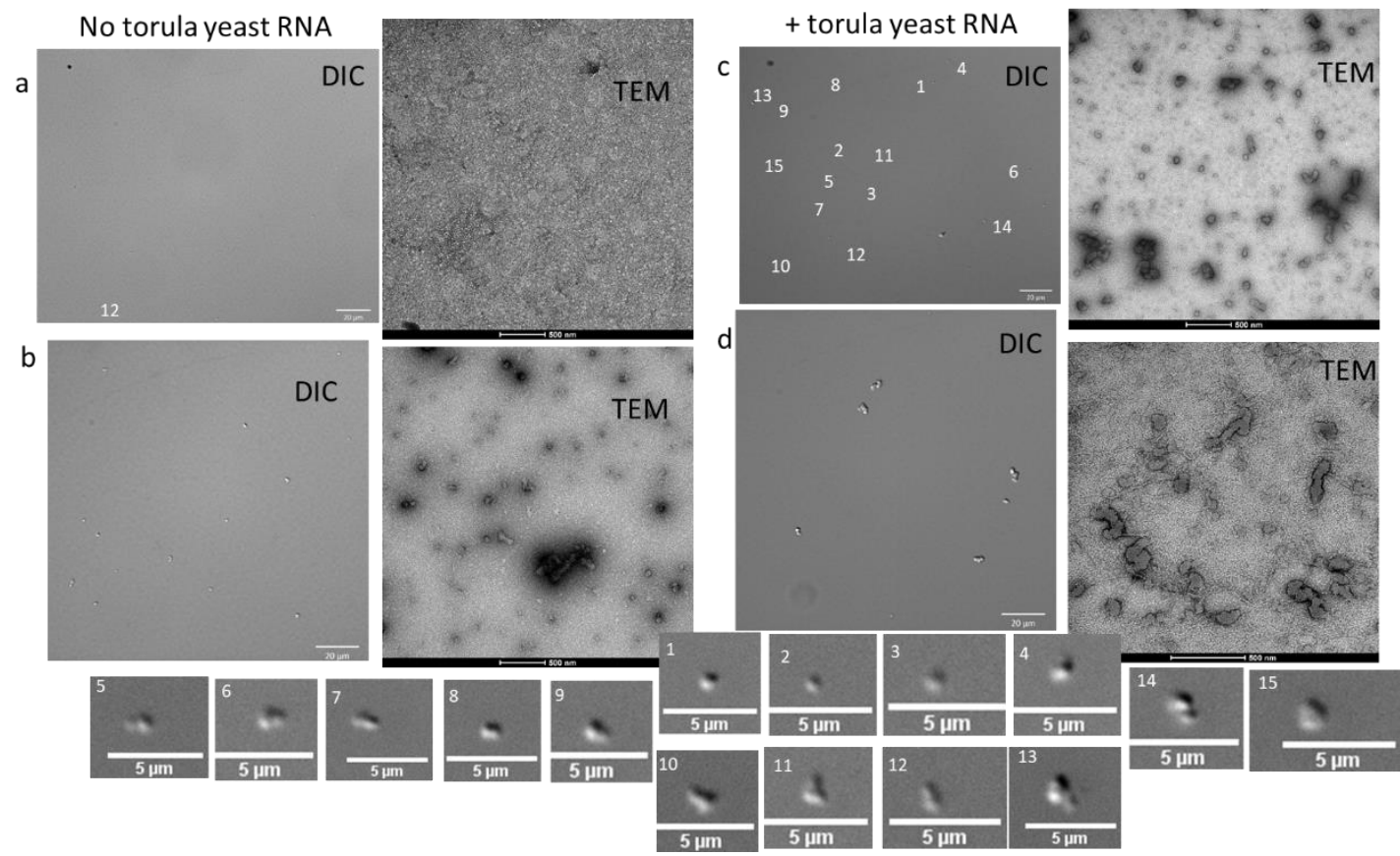

Figure S3. Growth of the TDP-43(311-360) fibrils monitored by fluorescence spectroscopy. The sample concentration was $186.8 \mu \mathrm{M}$ at the beginning of the dialysis. (a) The ThT fluorescence changes at $480 \mathrm{~nm}$ with the increasing amount of TDP- 
43(311-360) fibrils during dialysis. The ThT concentration was $10 \mu \mathrm{M}$ in the fluorescence measurement. (b) The protein intrinsic fluorescence changes during the same process. The fluorescence maximum wavelength changed after $1 \mathrm{~h}$ of dialysis (inset). These are repeat experiments, shown the similar results as in Figure 2.
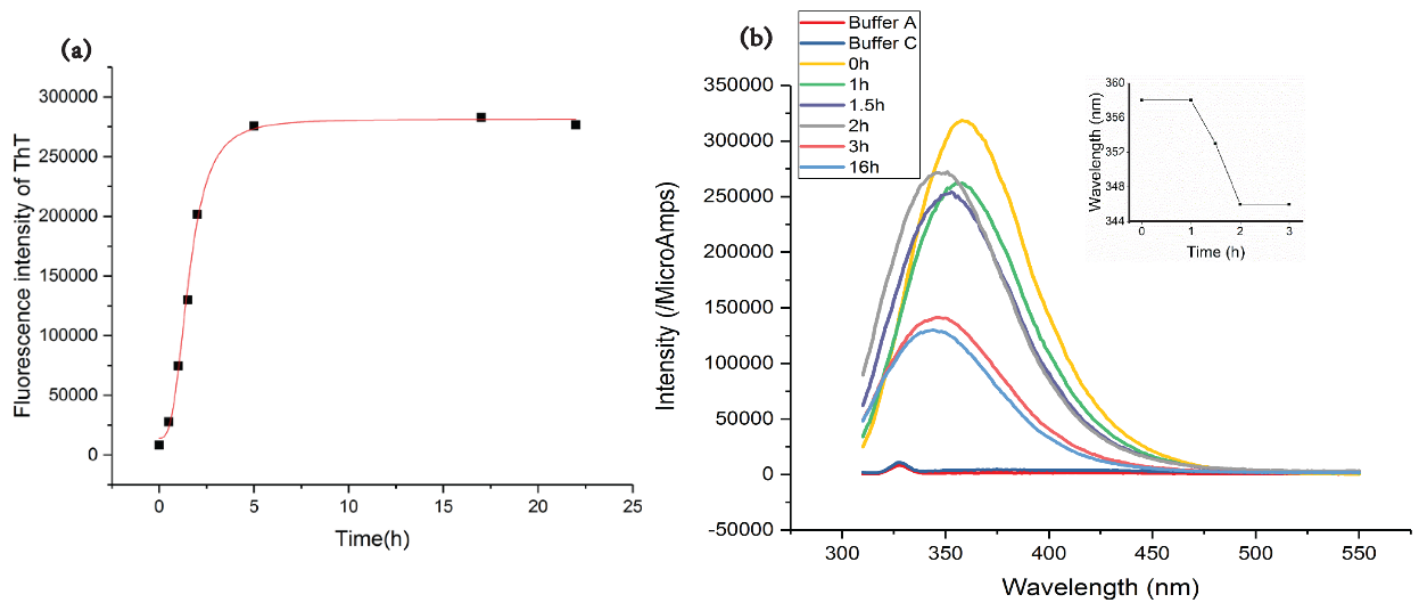

Figure S4. NCOCX spectrum of the uniformly $\left[{ }^{13} \mathrm{C},{ }^{15} \mathrm{~N}\right]$-labeled TDP-43 (311-360) fibrils. The backbone nitrogen positions of $\mathrm{Q} 343$ and $\mathrm{Q} 344$ and $\mathrm{N} \varepsilon 2$ of $\mathrm{Q} 343$ are underlined. 


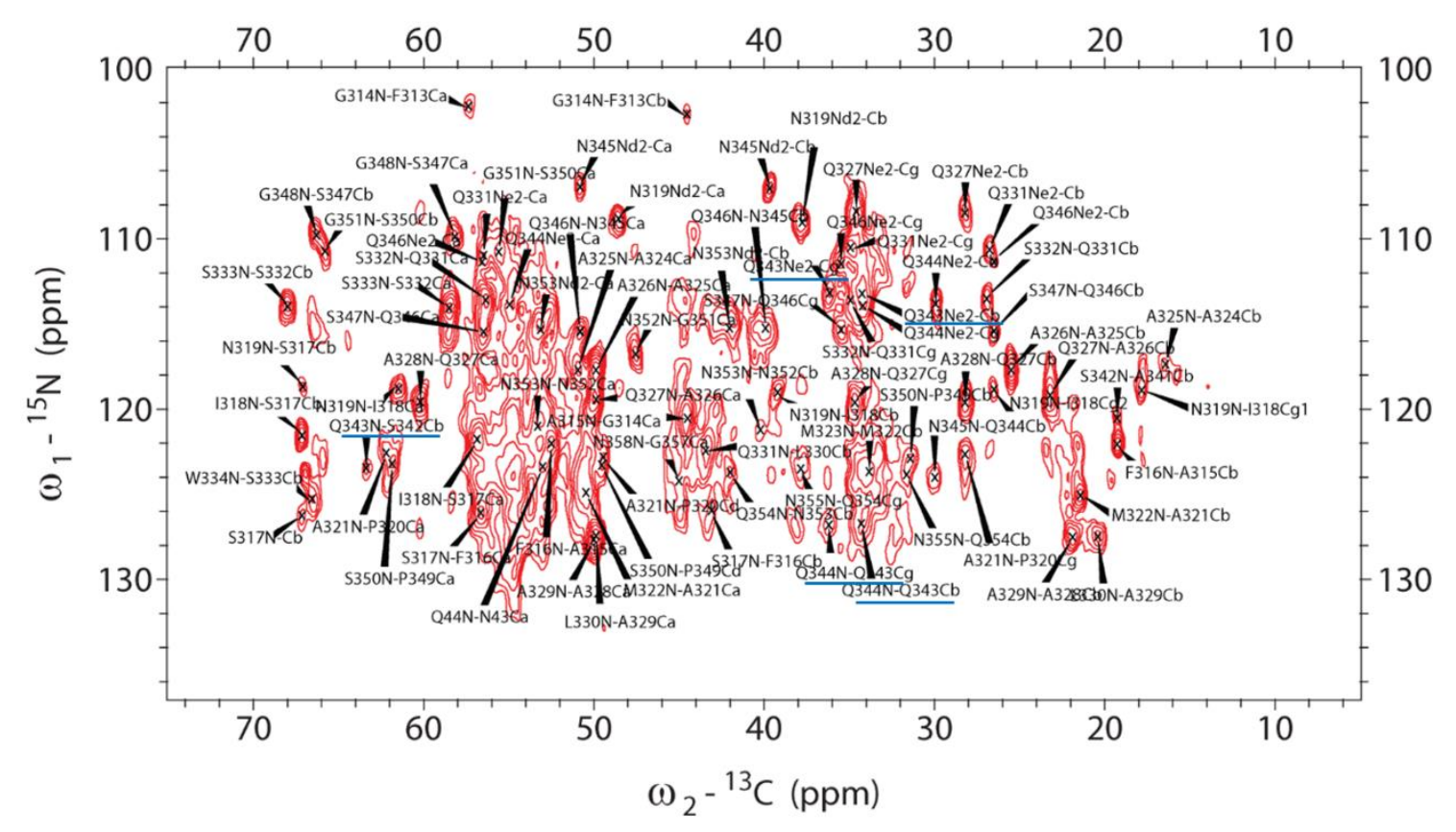

Figure S5. Plot of the difference in the secondary chemical shifts between $\mathrm{C} \alpha$ and $\mathrm{C} \beta$, calculated using the formula: $\Delta \mathrm{C} \alpha$ $\Delta C \beta=\{\mathrm{C} \alpha(\mathrm{obs})-\mathrm{C} \alpha(\mathrm{rc})\}-\{\mathrm{C} \beta(\mathrm{obs})-\mathrm{C} \beta(\mathrm{rc})\} . \mathrm{C} \alpha(\mathrm{obs})$ and $\mathrm{C} \beta$ (obs) are the observed chemical shifts from the experiments, $\mathrm{C} \alpha(\mathrm{rc})$ and $\mathrm{C} \beta(\mathrm{rc})$ are the chemical shifts for the random coil structure obtained from BMRB (biological magnetic resonance bank, http://www.bmrb.wisc.edu/ref_info/). 


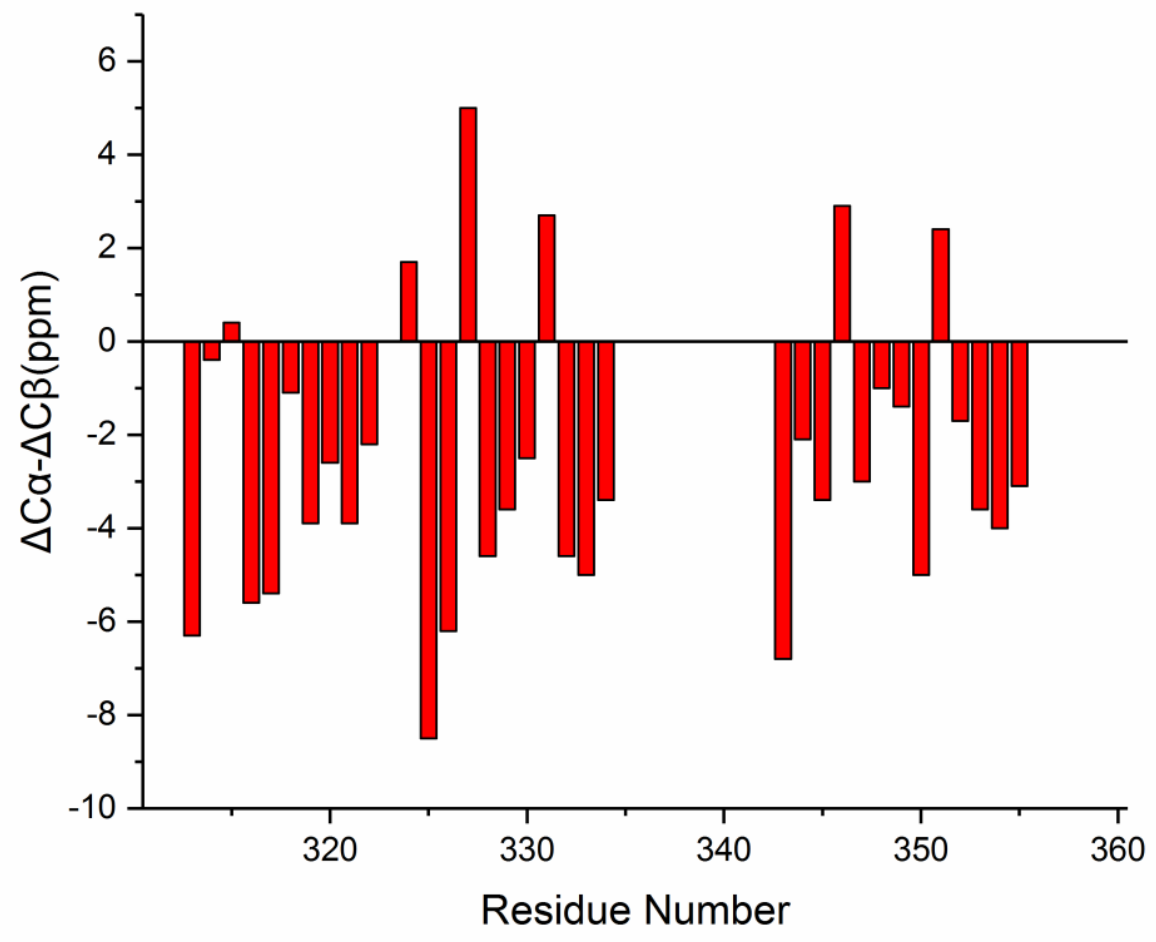

Figure $\mathrm{S} 6.2 \mathrm{D}^{13} \mathrm{C}-{ }^{13} \mathrm{C}$ correlation spectrum using DARR with a mixing time of $500 \mathrm{~ms}$, showing (a) Ala $\mathrm{C} \beta$ correlation peaks and (b) ${ }^{15} \mathrm{~N}-{ }^{13} \mathrm{C}$ TEDOR spectrum of sparsely ${ }^{13} \mathrm{C}$-labeled TDP-43 (311-360) fibrils using 1,3- ${ }^{13} \mathrm{C}$-glycerol. The cross peak of $A 329 C \beta-A 326 C \beta$ is highlighted with a rectangle in (a) and the corresponding $C \beta$ peaks connected to TEDOR are underlined in (b). 


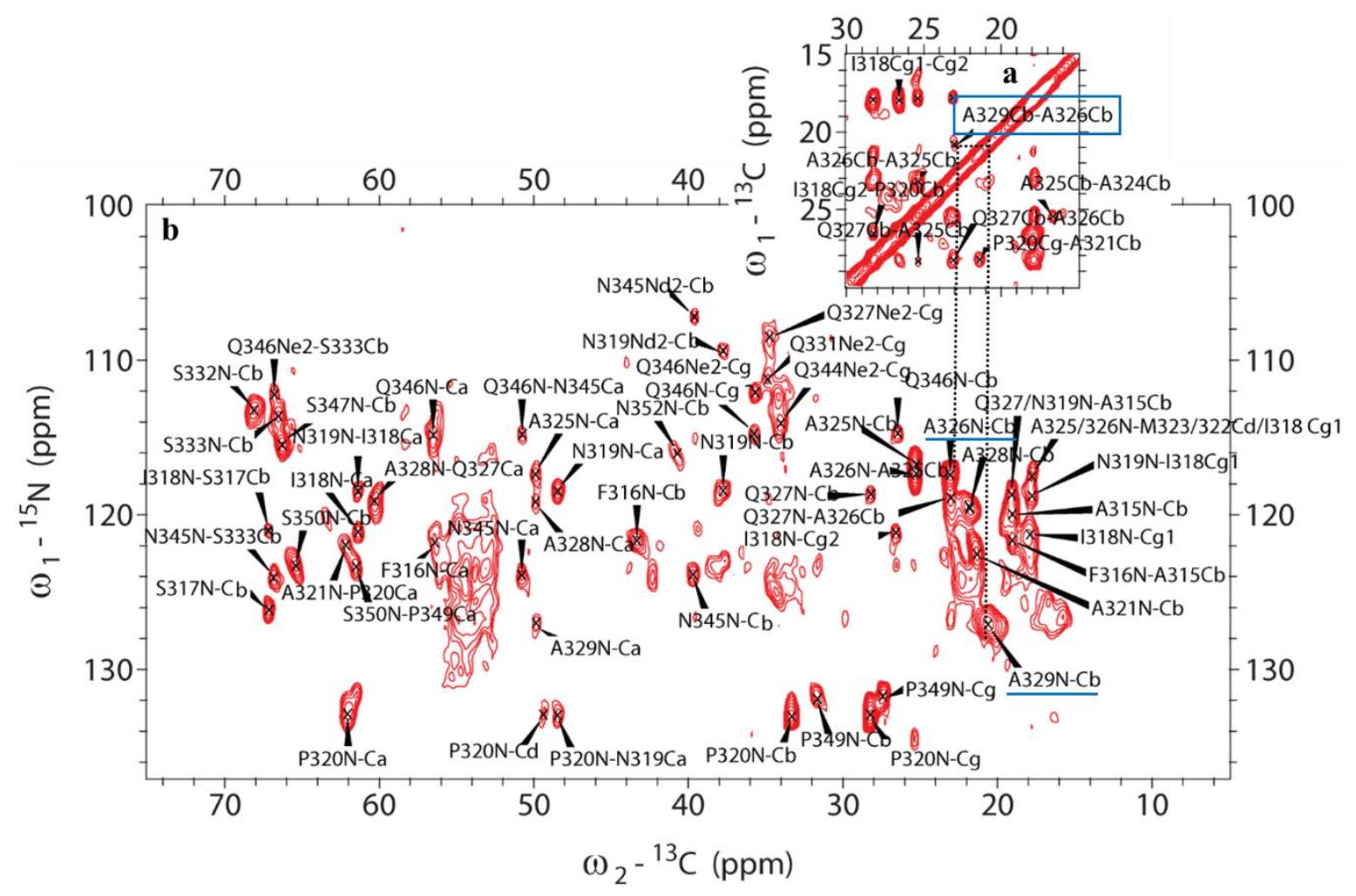

Figure S7. J-coupling based ${ }^{13} \mathrm{C}^{13} \mathrm{C}$ TOBSY spectrum of the uniformly ${ }^{13} \mathrm{C}-$ labeled TDp-43 (311-360) fibrils. 


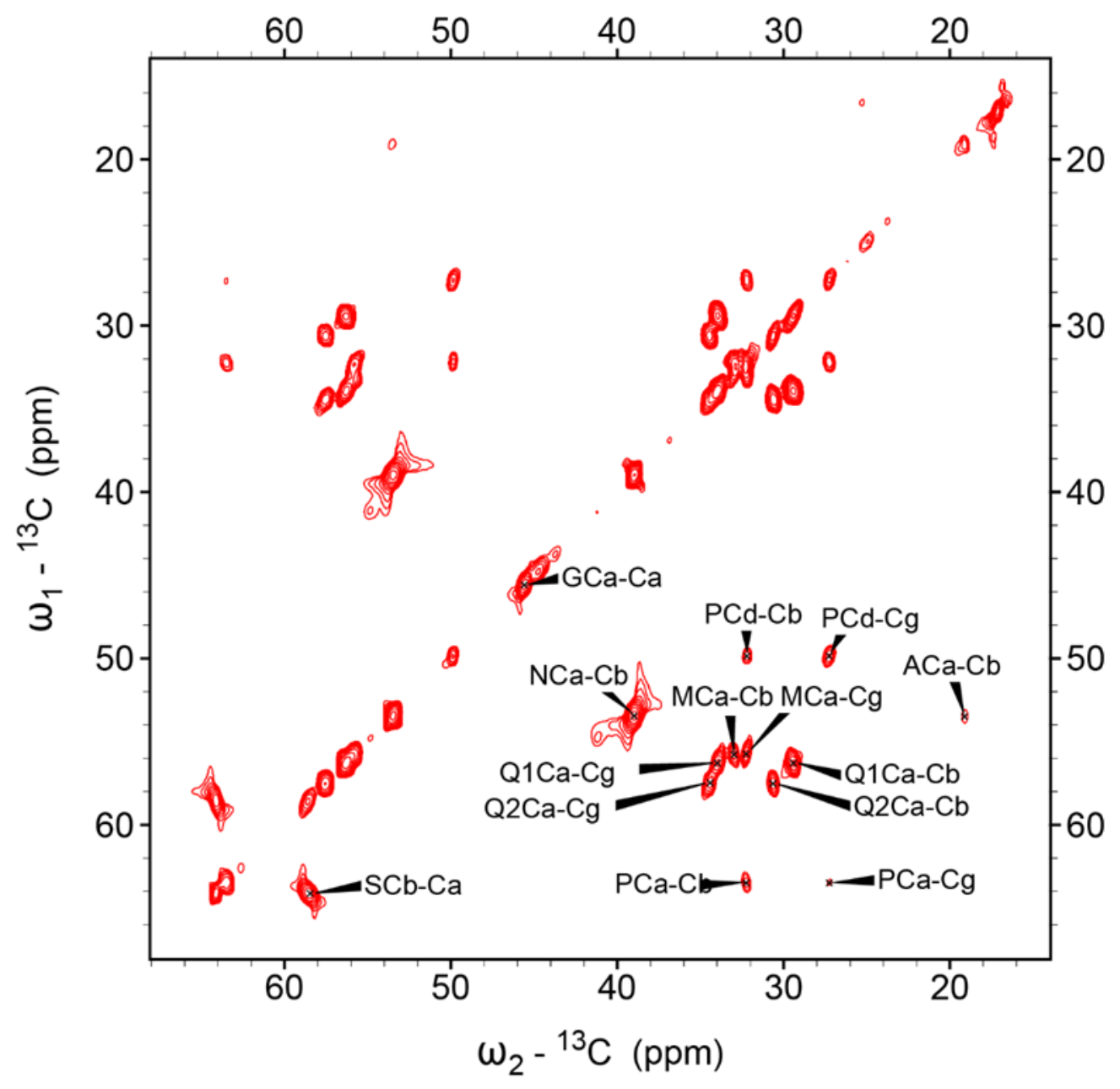

